\title{
Robust approximations for pricing Asian options and volatility swaps under stochastic volatility
}

\author{
Martin Forde* Antoine Jacquier ${ }^{\dagger}$
}

\begin{abstract}
We show that if the discounted Stock price process is a continuous martingale, then there is a simple relationship linking the variance of the terminal Stock price and the variance of its arithmetic average. We use this to establish a model-independent upper bound for the price of a continuously sampled fixed-strike Arithmetic Asian call option, in the presence of non-zero time-dependent interest rates (Theorem 1.2). We also propose a model-independent lognormal moment-matching procedure for approximating the price of an Asian call, and we show how to apply these approximations under the Black-Scholes and Heston models (subsection 1.3). We then apply a similar analysis to a time-dependent Heston stochastic volatility model, and we show to construct a time-dependent mean reversion and volatility-of-variance function, so as to be consistent with the observed variance swap curve and a pre-specified term structure for the variance of the integrated variance (Theorem 2.1). We characterize the small-time asymptotics of the first and second moments of the integrated variance (Proposition 2.2), and derive an approximation for the price of a volatility swap under the time-dependent Heston model (Eq (52)), using the Brockhaus-Long approximation[BL00]. We also outline a bootstrapping procedure for calibrating a piecewise-linear mean reversion level and volatilityof-volatility function (subsection 2.3.2).
\end{abstract}

\section{Introduction}

Under the Black-Scholes model, there is no closed-form solution for the price of an Arithmetic Asian option. Geman\&Yor[GY93] proposed a methodology using time horizon Laplace transforms and properties of time-changed Bessel processes, but their approach only works when the risk-neutral drift is not less than half the squared volatility. This restriction was lifted by Carr\&Schroder[CS00] using complex analytic techniques. Donati-Martin,Ghomasni\&Yor[DGY01] use time inversion applied to the Brownian exponential functional to show that it has the same law as a certain diffusion process. Levy[Levy92] approximated the price of an Asian option by fitting a lognormal distribution to the Arithmetic Average. Posner\&Milevsky[PM98] extended Levy's approach to allow for the exact fitting of the third and fourth moments to a Johnson distribution. In another article, Milevsky\&Posner [1998] show that, under certain conditions, when the amount of fixings and

\footnotetext{
*Department of Mathematical Sciences, Dublin City University, Glasnevin, Dublin 9, Ireland (Martin.Forde@dcu.ie), work supported by SFI grant for the Edgeworth Centre for Financial Mathematics and European Science Foundation, AMaMeF Exchange Grant 2107.

${ }^{\dagger}$ Imperial College, 180 Queen's Gate, Department of Mathematics, London and Zeliade Systems, 56 Rue JeanJacques Rousseau, Paris (antoine.jacquier08@imperial.ac.uk).
} 
the time to maturity approach infinity, the law of the arithmetic average approaches an inverse gamma distribution. Rogers\&Shi[RS95] derive a sharp lower bound using a general inequality for conditional expectations of a call option payoff, and conditioning on the arithmetic average of the driving Brownian motion; the distribution of the log Stock price conditioned on this random variable is known and normally distributed. Thompson[T99] derives an upper bound which sharpens the upper bound which appears in Rogers\&Shi[RS95].

In this article, we take a non-parametric approach. We assume only that the discounted Stock price process is a non-negative continuous martingale. Under this assumption, we show how to extract the first and second moments of the arithmetic average Stock price, without any further model specification, in terms of the variance of the terminal Stock prices (Theorem 1.1), which we can extract from the observed prices of European options. Using this information, we then establish a robust upper bound for the price of an Asian call, by pricing any quadratic payoff on the average Stock price which dominates the Asian call payoff, and then choosing the cheapest possible payoff function amongst all such quadratics (Theorem 1.2). We show that Theorems 1.1 and 1.2 can be applied to the Black-Scholes and Heston models, and in both cases we present closed-form formulae for the variance of the arithmetic average Stock price. We also propose a lognormal moment-matching approximation (subsection 1.4), which is similar in spirit to the methodology in Levy[Levy92], but here we are using the moments that are implied by European option prices rather than a parametric Black-Scholes model.

In section 3 we propose an extension of the well known Heston stochastic volatility model, with a time-dependent mean reversion level $\theta(t)$, and time-dependent volatility-of-variance $\sigma(t)$. This model has also been discussed in Buehler[Buehler06]. Proceeding along similar lines to Dufresne[Duf01] and Gatheral[Gath07], we show how construct $\theta(t)$ which is consistent with an observed or pre-specified variance swap curve, and a $\sigma(t)$ which is consistent with a pre-specified term structure for the variance of the integrated variance. We discuss how to combine this methodology with the Carr-Lee[CL08] correlation-neutral formula for the second moment of the integrated variance, expressed in terms the price of a European style contract on the underlying Stock. We also derive Taylor series expansions for these quantities in the maturity variable and propose a bootstrapping algorithm for calibrating a piecewise linear $\theta(t)$ and $\sigma(t)$. Finally, we derive a smalltime estimate for the volatility swap rate $(\mathrm{Eq}(52))$ by combining these Taylor series with the Brockhaus-Long[BL00] approximation.

\section{Robust bounds and approximations for Asian options}

\subsection{Computing the variance of the average Stock price from the variance of the Stock price}

Throughout this section, we work on a probability space $(\Omega, \mathcal{F}, \mathbb{P})$ endowed with a filtration $\left\{\mathcal{F}_{t}\right\}$, which satisfies the usual conditions of definition 1.2.25 in Karatzas\&Shreve[KS91], and we assume that $\mathbb{P}$ is a risk-neutral measure.

Theorem 1.1 Consider the following stochastic volatility model for a Stock price process $S$

$$
\begin{aligned}
S_{t} & =e^{\int_{0}^{t} r(u) d u} \tilde{S}_{t}, \\
d \tilde{S}_{t} & =\tilde{S}_{t} \sigma_{t} d W_{t}
\end{aligned}
$$


under $\mathbb{P}$, where $W$ is a standard Brownian motion. We assume that $\tilde{S}_{t}$ is a non-negative, continuous martingale and $\sigma_{t}$ is a progressively measurable non-negative volatility process. $r(t)$ is a time-dependent interest rate with $0 \leq r(t)<r_{\max }<\infty$ for all $t$, and we write $B_{t}=e^{\int_{0}^{t} r(u) d u}$. Then we have the following relationship between the second moments of $S_{t}$ and the second moment of $\int_{0}^{t} S_{u} d u$

$$
\mathbb{E}\left(\left(\int_{0}^{t} S_{u} d u\right)^{2}\right)=2 \int_{0}^{t} B_{s} \int_{0}^{s} B_{u}^{-1} \mathbb{E}\left(S_{u}^{2}\right) d u d s
$$

Proof. Let $I_{t}=\int_{0}^{t} S_{u} d u$. $I_{t}$ is a continuous semimartingale, so we can apply Itô's lemma and take the expectation to obtain

$$
\mathbb{E}\left(I_{t}^{2}\right)=2 \mathbb{E}\left(\int_{0}^{t} I_{u} S_{u} d u\right)
$$

By Fubini's theorem and taking conditional expectations, we can re-write this expression as

$$
\begin{aligned}
\mathbb{E}\left(I_{t}^{2}\right) & =2 \int_{0}^{t} \mathbb{E}\left(I_{u} S_{u}\right) d u \\
& =2 \int_{0}^{t} \int_{0}^{u} \mathbb{E}\left(S_{u} S_{v}\right) d v d u \\
& =2 \int_{0}^{t} \int_{0}^{u} \mathbb{E}\left(S_{v} \mathbb{E}_{v} S_{u}\right) d v d u \\
& =2 \int_{0}^{t} B_{u} \int_{0}^{u} B_{v}^{-1} \mathbb{E}\left(S_{v}^{2}\right) d v d u .
\end{aligned}
$$

\subsection{A model-independent upper bound for the price of an Asian call option}

Theorem 1.2 Under the assumptions of Theorem 1.1, we have the following upper bound for the price $P$ of a $K$-strike continuously-sampled arithmetic Asian call option which pays $\left(I_{t}-K\right)^{+}$at time $t$

$$
P=B_{t}^{-1} \mathbb{E}\left(I_{t}-K\right)^{+} \leq \frac{B_{t}^{-1}}{4\left(K-L^{*}\right)}\left[\mathbb{E}\left(I_{t}^{2}-2 L^{*} \mathbb{E}\left(I_{t}\right)+L^{* 2}\right],\right.
$$

where

$$
L^{*}=K-\left[\operatorname{Var}\left(I_{t}\right)+\left(K-\mathbb{E}\left(I_{t}\right)\right)^{2}\right]^{\frac{1}{2}},
$$

and we can use Theorem 1.1 to write the value of $\mathbb{E}\left(I_{t}^{2}\right)$ in terms of $\mathbb{E}\left(S_{u}^{2}\right)$ for $u \in[0, t]$.

Remark 1.1 If we can observe European option prices at all strikes and all maturities in $[0, t]$, then we can back out $\mathbb{E}\left(S_{u}^{2}\right)$ for all $u \in[0, t]$ using the well known Breeden-Litzenberger[BL78] result, and then use this information to compute the Variance of $\int_{0}^{t} S_{u} d u$ that is implied by the market. We can then plug this value for the Variance into the upper bound in Theorem 1.2. 
Proof. Theorem 1.1 implies that we can compute $\mathbb{E}\left(\int_{0}^{t} S_{u} d u\right)$ and $\mathbb{E}\left(\int_{0}^{t} S_{u} d u\right)^{2}$ if we know the Variance of $S_{u}$ for all $u \in[0, t]$. Thus we can price any quadratic payoff $\mathrm{Q}\left(I_{t}\right)$ on $I_{t}=\int_{0}^{t} S_{u} d u$. Consider the following choice of $Q($.

$$
\mathrm{Q}(I)=\frac{1}{4(K-L)}(I-L)^{2} .
$$

with $0 \leq L<K . Q(I)$ is always greater than or equal to $(I-K)^{+}$, and touches $(I-K)^{+}$at $I=L$, and $I=U=2 K-L>K$, so we have:

$$
\begin{aligned}
\mathbb{E}\left(I_{t}-K\right)^{+} & \leq \mathbb{E}\left(\mathrm{Q}\left(I_{t}\right)\right) \\
& =\frac{1}{4(K-L)}\left[\mathbb{E}\left(I_{t}^{2}\right)-2 L \mathbb{E}\left(I_{t}\right)+L^{2}\right] .
\end{aligned}
$$

We now wish to find the value of $L$ that minimizes the value of $\mathbb{E}\left(\mathrm{Q}\left(I_{t}\right)\right)$. Differentiating with respect to $L$, and setting the derivative to zero we obtain

$$
\begin{aligned}
\frac{\partial}{\partial L} \mathbb{E}\left(\mathrm{Q}\left(I_{t}\right)\right) & =\frac{2 L-2\left(I_{t}\right)}{4(K-L)}+\frac{\mathbb{E}\left(I_{t}^{2}\right)-2 L \mathbb{E}\left(I_{t}\right)+L^{2}}{4(K-L)^{2}} \\
& =\frac{2\left(L-\mathbb{E}\left(I_{t}\right)\right)(K-L)+\mathbb{E}\left(I_{t}^{2}-2 L I_{t}+L^{2}\right)}{4(K-L)^{2}} \\
& =0 .
\end{aligned}
$$

Finally, solving the quadratic in $L$ in the numerator, we see that the relevant root, and thus the optimal value of $L$, is given by

$$
L^{*}=K-\left[\operatorname{Var}\left(I_{t}\right)+\left(K-\mathbb{E}\left(I_{t}\right)\right)^{2}\right]^{\frac{1}{2}}=K-\left[\mathbb{E}\left(I_{t}-K\right)^{2}\right]^{\frac{1}{2}} .
$$

Remark 1.2 As we would expect by drawing the graph of $\mathrm{Q}, L^{*}$ is lower, and its corresponding $U^{*}$ is higher, when the variance of $I_{t}$ is higher, and when the Asian call option is further out-ofthe-money (see graph below).

\subsection{Examples}

\subsubsection{The Black Scholes model}

For the Black Scholes model

$$
d S_{t}=r S_{t} d t+\sigma S_{t} d W_{t},
$$

the discounted Stock price $\tilde{S}_{t}=e^{-r t} S_{t}$ is a martingale, and $\mathbb{E}\left(S_{t}^{p}\right)<\infty$ for all $p>1$. Thus we are within the jurisdiction of Theorem 1.1, and the formula in Eq (2) reduces to the following expression

$$
\begin{aligned}
\mathbb{E}\left(\int_{0}^{t} S_{u} d u\right)^{2} & =\frac{2 S_{0}^{2}}{t^{2}}\left(\frac{e^{\left(2 r+\sigma^{2}\right) t}}{\left(r+\sigma^{2}\right)\left(2 r+\sigma^{2}\right)}+\frac{1}{r}\left(\frac{1}{2 r+\sigma^{2}}-\frac{e^{r t}}{r+\sigma^{2}}\right)\right) \text { for } r>0, \\
& =\frac{2 S_{0}^{2}}{t^{2}}\left(\frac{e^{\sigma^{2} t}-1-\sigma^{2} t}{\sigma^{4}}\right) \quad \text { for } r=0,
\end{aligned}
$$

which also appears in Milevsky\&Posner[MP98]. 


\subsubsection{The Heston model}

Consider the well known Heston model defined by the following stochastic differential equations

$$
\begin{aligned}
d S_{t} & =r S_{t} d t+S_{t} \sqrt{v_{t}} d W_{t}, \\
d v_{t} & =\kappa\left(\theta-v_{t}\right) d t+\sigma \sqrt{v_{t}} d B_{t},
\end{aligned}
$$

with $d\langle W, B\rangle_{t}=\rho d t, \kappa, \theta, \sigma, v_{0}>0,|\rho|<1$. The discounted Stock price process $\tilde{S}_{t}=e^{-r t} S_{t}$ is a Martingale (see Proposition 2.5 in Andersen\&Piterbarg[AP07]). We can compute $\mathbb{E}\left(S_{t}^{2}\right)$ in closedform using the analytic continuation of the well known analytic exponential affine characteristic function for the log Stock price (see Heston[Hest93] for a PDE proof using Riccati equations, or Hurd\&Kuznetsov[HK08] for a probabilistic treatment). From Eq (2), we find that

$$
\begin{aligned}
\mathbb{E}\left(\left(\int_{0}^{t} S_{u} d u\right)^{2}\right) & =2 \int_{0}^{t} e^{r s} \int_{0}^{s} e^{-r u} \mathbb{E}\left(S_{u}^{2}\right) d u d s \\
& =S_{0}^{2} \int_{0}^{t} e^{r s} \int_{0}^{s} e^{-r u} \frac{e^{\frac{(\kappa-2 \rho \sigma) \kappa \theta u}{\sigma^{2}}} e^{\frac{2 v_{0}}{\kappa-2 \rho \sigma+\gamma \operatorname{coth}\left(\frac{1}{2} \gamma u\right)}}}{\left(\cosh \left(\frac{1}{2} \gamma u\right)+\frac{\kappa-2 \rho \sigma}{\gamma} \sinh \left(\frac{1}{2} \gamma u\right)\right)^{\frac{2 \kappa \theta}{\sigma^{2}}}} d u d s \\
& =S_{0}^{2}\left(t^{2}+\frac{1}{3}\left(r+v_{0}\right) t^{3}+\frac{1}{12}\left(r^{2}+r v_{0}+\kappa\left(\theta-v_{0}\right)+v_{0}^{2}+2 v_{0} \sigma \rho\right) t^{4}+\mathcal{O}\left(t^{5}\right)\right),
\end{aligned}
$$

where $\gamma=\sqrt{(\kappa-2 \rho \sigma)^{2}-2 \sigma^{2}}$. For $v_{0}=\theta$ and $r=\rho=0$, we have

$$
\mathbb{E}\left(\left(\int_{0}^{t} S_{u} d u\right)^{2}\right)=S_{0}^{2}\left(t^{2}+\frac{1}{3} v_{0} t^{3}+\frac{1}{12} v_{0}^{2} t^{4}+\frac{1}{60}\left(v_{0}^{3}+v_{0} \sigma^{2}\right) t^{5}+\mathcal{O}\left(t^{6}\right)\right) .
$$

We see that the volatility-of-variance term $\sigma$ only appears on its own at $\mathcal{O}\left(t^{5}\right)$.

\subsection{Approximating the price of an Asian option with lognormal Moment- matching}

We can proceed along similar lines to Levy[Levy92] and Posner\&Milevsky[PM98] by matching the first and second moments of $\int_{0}^{t} S_{u} d u$ to a lognormal distribution. We can then approximate the price of an Asian call option as if $X=\log \int_{0}^{t} S_{u} d u \sim N\left(\mu t, \sigma^{2} t\right)$, by solving the following two equations

$$
\begin{aligned}
\mathbb{E}\left(e^{X}\right) & =e^{\left(\mu+\frac{1}{2} \sigma^{2}\right) t}, \\
\mathbb{E}\left(e^{2 X}\right) & =e^{2\left(\mu+\sigma^{2}\right) t} .
\end{aligned}
$$

for $\mu$ and $\sigma$, and then using the Black-Scholes formula to approximate Asian call prices. Recall Remark 1.1. 


\subsection{Numerical results}

In the table and Figure 1.5 below, we have tabulated and plotted the prices of ten continuously monitored Arithmetic Asian call options under the Black Scholes model $d S_{t}=r S_{t} d t+\sigma S_{t} d W_{t}$, with $r=0, \sigma=.10$ and $T=1$ year maturity, and compared with the Moment-matching approximation and the upper bound in Eq (5). The Monte Carlo prices and the Moment Matched prices are virtually indistinguishable, and we see that the upper bound becomes less useful for Asian options which are heavily out-of-the-money. The true standard deviation of the arithmetic average Stock price is $5.78 \%$ (using Eq (12) ), which is approximately .10/ $\sqrt{3}=5.77 \%$. We used an Euler Monte Carlo scheme with 100 time steps and 1,000,000 simulations.

\begin{tabular}{|c|c|c|c|c|c|}
\hline$K$ & Monte Carlo & $L^{*}$ & $U^{*}$ & Upper bound & Moment-Matching \\
\hline 0.90 & 0.1007 & 0.7845 & 1.0155 & 0.1078 & 0.1007 \\
\hline 0.92 & 0.0818 & 0.8213 & 1.0187 & 0.0893 & 0.0818 \\
\hline 0.94 & 0.0641 & 0.8597 & 1.0233 & 0.0717 & 0.0641 \\
\hline 0.96 & 0.0480 & 0.8897 & 1.0303 & 0.0551 & 0.0480 \\
\hline 0.98 & 0.0343 & 0.9188 & 1.0412 & 0.0406 & 0.0342 \\
\hline 1.00 & 0.0232 & 0.9422 & 1.0578 & 0.0289 & 0.0230 \\
\hline 1.02 & 0.0148 & 0.9588 & 1.0812 & 0.0206 & 0.0146 \\
\hline 1.04 & 0.0089 & 0.9697 & 1.1103 & 0.0151 & 0.0087 \\
\hline 1.06 & 0.0051 & 0.9767 & 1.1433 & 0.0117 & 0.0049 \\
\hline 1.08 & 0.0027 & 0.9813 & 1.1787 & 0.0093 & 0.0025 \\
\hline 1.10 & 0.0014 & 0.9845 & 1.2155 & 0.0078 & 0.0012 \\
\hline
\end{tabular}




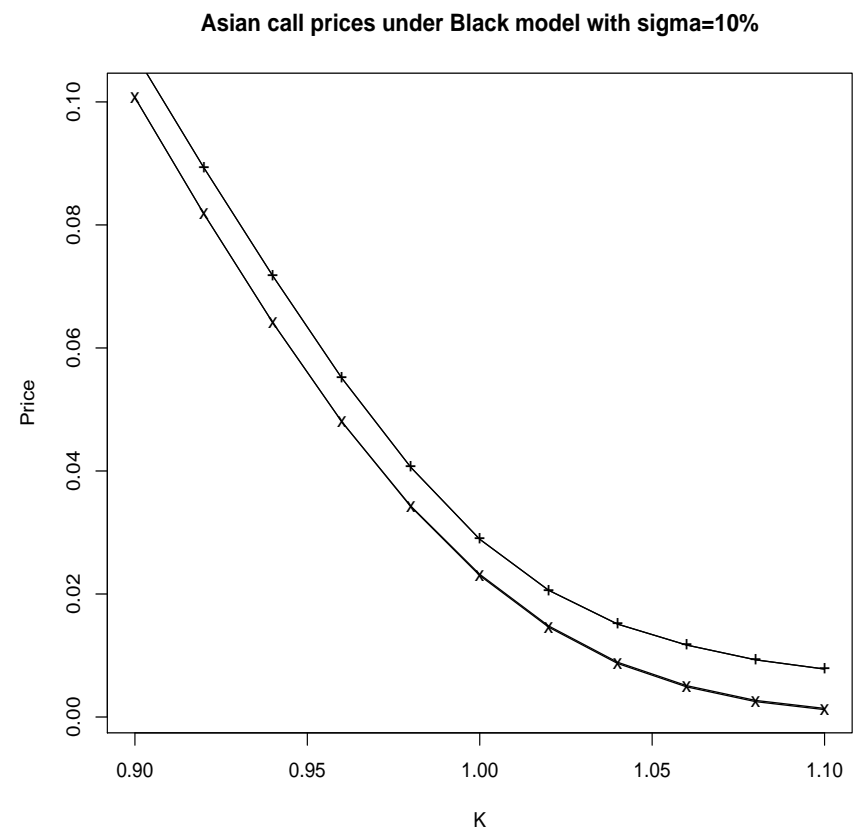

$Q(A)$ for Asian call with $K=1.04$ under Black model with sigma=10\%

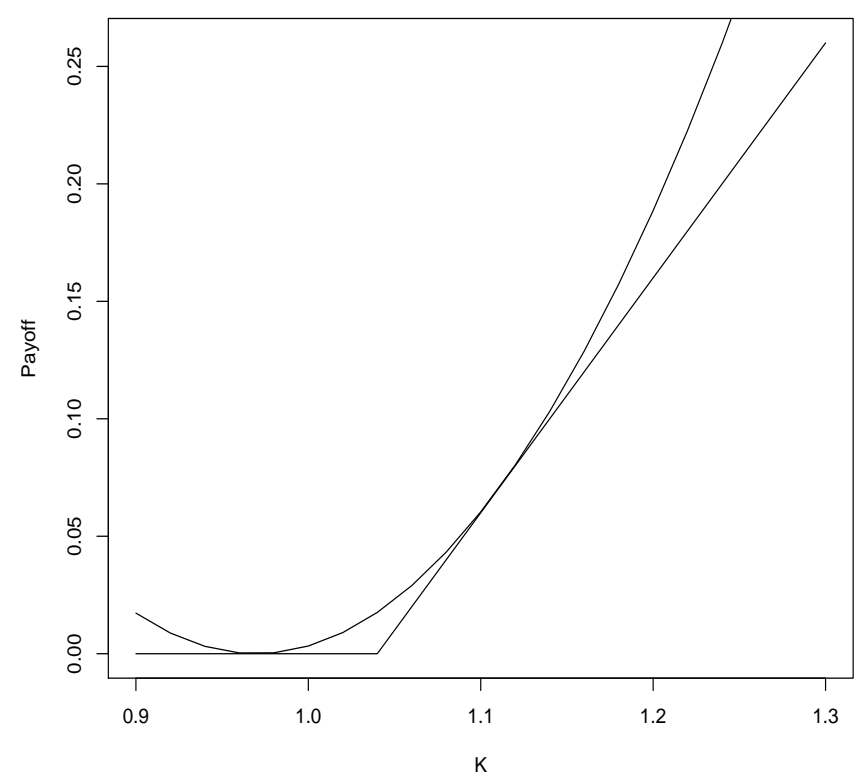

Figure 1: Here we have plotted the optimal quadratic payoff function for the parameters given in the title of the plot. 


\section{Calibrating a time-dependent Heston model and pricing volatility swaps}

\section{$2.1 \quad$ Introduction}

For a Stock price process $S$ which is a positive continuous martingale under the pricing measure, we can write

$$
S_{t}=S_{0} \mathcal{E}(X)=S_{0} e^{X_{t}-\frac{1}{2}\langle X\rangle_{t}},
$$

where

$$
X_{t}=\int_{0}^{t} \sqrt{v_{s}} d W_{s}
$$

for some Brownian motion $W$ and a predictable square integrable volatility process $\sqrt{v_{t}}$ (see Buehler[Buehler06]). For the process $S$, the fair price of a variance swap (at time zero) is $\mathbb{E}_{0}\left(\int_{0}^{t} v_{s} d s\right)$. The variance swap can be replicated with log contracts on $S_{t}$, which can in turn be replicated with a static portfolio of European call and put options of the same maturity $t$ (see Neuberger[Neu92] and Appendix A of Buehler[Buehler06] for details).

Brockhaus\&Long[BL00] used a second order truncated Taylor series expansion to derive the following approximation

$$
\mathbb{E}\left(\sqrt{\langle X\rangle_{t}}\right) \approx \sqrt{\mathbb{E}\left(\langle X\rangle_{t}\right)}-\frac{\operatorname{Var}\left(\langle X\rangle_{t}\right)}{8 \mathbb{E}\left(\langle X\rangle_{t}\right)^{\frac{3}{2}}}
$$

We define the initial variance swap curve $\operatorname{VAR}_{0}(t)$ and the volatility swap curve $\operatorname{VoL}_{0}(t)$ as follows

$$
\begin{aligned}
& \operatorname{VAR}_{0}(t)=\sqrt{\mathbb{E}\left(\langle X\rangle_{t}\right)}, \\
& \operatorname{VoL}_{0}(t)=\mathbb{E}\left(\sqrt{\langle X\rangle_{t}}\right)
\end{aligned}
$$

$(\operatorname{VoL}(t) \leq \operatorname{VAR}(t)$ by Jensen's inequality). We have made no assumptions about the correlation structure between $W$ and $v$. Under a general stochastic volatility framework where the correlation $\rho$ between the two Brownian motions is small, Carr\&Lee[CL08] derived the following correlationneutral approximation

$$
\mathbb{E}\left(\langle X\rangle_{t}^{2}\right) \approx \mathbb{E}\left(4 X_{t}^{2}+16 X_{t}+8 X_{t} e^{X_{t}}-24 e^{X_{t}}+24\right)
$$

for the second moment of the integrated variance, in terms of the price of a European style contract paying $4 X_{t}^{2}+16 X_{t}+8 X_{t} e^{X_{t}}-24 e^{X_{t}}+24$ (see section 7.2 in [CL08]). When the correlation is zero, this approximation becomes an equality, and in this special case Carr\&Lee[CL08] also derived the following bounds

$$
\frac{2 \sqrt{\pi}}{S_{0}} \mathbb{E}\left(S_{t}-S_{0}\right)^{+} \leq \mathrm{IV}_{0}(t) \leq \operatorname{VOL}_{0}(t) \leq \operatorname{VAR}_{0}(t)=-2 \mathbb{E}\left(\log \left(\frac{S_{t}}{S_{0}}\right)\right),
$$

where $\mathrm{IV}_{0}(t)$ is the unannualized At-the-Money implied volatility for a European call option of maturity $t$, and the following approximation for the volatility swap rate

$$
\mathrm{VOL}_{0}(\mathrm{t}) \approx \mathrm{IV}_{0}(t)\left(1+\frac{\mathrm{VAR}_{0}(t)^{2}-\mathrm{IV}_{0}^{2}(t)}{8+2 \mathrm{IV}_{0}^{2}(t)}\right)
$$




\subsection{Calibrating a time-dependent Heston model}

Using a similar analysis to Theorem 1.1, we now construct an extension of the standard Heston stochastic volatility model, with time-dependent mean reversion level and volatility-of-variance, so as to be consistent with an observed or pre-specified variance swap curve, and a pre-specified term structure for the second moment of the integrated variance.

Theorem 2.1 Consider the following extension of the stochastic volatility model introduced by Heston[Hest93], defined by the following stochastic differential equations

$$
\begin{aligned}
d S_{t} & =r S_{t} d t+S_{t} \sqrt{v_{t}} d W_{t} \\
d v_{t} & =\kappa\left(\theta(t)-v_{t}\right) d t+\sigma(t) \sqrt{v_{t}} d B_{t}
\end{aligned}
$$

with $d\langle W, B\rangle_{t}=\rho d t,|\rho|\left\langle 1, \kappa>0, v_{0}>0, \theta(t)>0, \sigma(t)>0\right.$. Then we have the following forward equations for $I_{t}=\int_{0}^{t} v_{s} d s$

$$
\begin{aligned}
\frac{\partial}{\partial t} \mathbb{E}\left(I_{t}^{2}\right) & =2 \mathbb{E}\left(I_{t} v_{t}\right) \\
\frac{\partial}{\partial t} \mathbb{E}\left(I_{t} v_{t}\right) & =\mathbb{E}\left(I_{t}\left(\kappa\left(\theta(t)-v_{t}\right)\right)+\mathbb{E}\left(v_{t}^{2}\right)\right. \\
\frac{\partial}{\partial t} \mathbb{E}\left(v_{t}^{2}\right) & =2 \mathbb{E}\left(v_{t} \kappa\left(\theta(t)-v_{t}\right)\right)+\sigma^{2}(t) \mathbb{E}\left(v_{t}\right) .
\end{aligned}
$$

Moreover, we can make this model consistent with a pre-specified variance swap curve $\mathbb{E}\left(\int_{0}^{t} v_{s} d s\right)$, and a pre-specified term structure for the second moment of the integrated variance $\mathbb{E}\left(\left(\int_{0}^{t} v_{s} d s\right)^{2}\right)$ by inverting these equations and choosing $v_{0}, \theta(t)$ and $\sigma(t)$ as follows

$$
\begin{aligned}
v_{0} & =\left.\frac{\partial}{\partial t} \mathbb{E}\left(\int_{0}^{t} v_{s} d s\right)\right|_{t=0} \\
\theta(t) & =\frac{\frac{\partial}{\partial t} \mathbb{E}\left(v_{t}\right)}{\kappa}+\mathbb{E}\left(v_{t}\right) \\
\sigma^{2}(t) & =\frac{\frac{\partial}{\partial t} \mathbb{E}\left(v_{t}^{2}\right)-2 \kappa \mathbb{E}\left(v_{t}\left(\theta(t)-v_{t}\right)\right)}{\mathbb{E}\left(v_{t}\right)},
\end{aligned}
$$

if $0<\theta_{\min } \leq \theta(.) \leq \theta_{\max }<\infty, 0<\sigma_{\min } \leq \sigma(.) \leq \sigma_{\max }<\infty$, where

$$
\begin{aligned}
\mathbb{E}\left(v_{t}^{2}\right) & =\frac{\partial}{\partial t} \mathbb{E}\left(I_{t} v_{t}\right)-\mathbb{E}\left(I_{t}\left(\kappa\left(\theta(t)-v_{t}\right)\right)\right. \\
\mathbb{E}\left(I_{t} v_{t}\right) & =\frac{1}{2} \frac{\partial}{\partial t} \mathbb{E}\left(I_{t}^{2}\right)
\end{aligned}
$$

Proof. The $v_{t}$ process satisfies the Yamada-Watanabe condition (see page 291, Proposition 2.13 in Karatzas\&Shreve[KS91]), so it admits a unique strong solution (adapted to the natural filtration of $\left.\left(B_{t}\right)\right)$. Integrating Eq 26, we have that

$$
v_{t}=v_{0}+\int_{0}^{t} \kappa\left(\theta(s)-v_{s}\right) d s+\sigma(s) \sqrt{v_{s}} d B_{s} .
$$


From appendix A, we know that $\mathbb{E}\left(v_{s}\right)<\infty$ for all $s \in[0, t]$. Thus $\mathbb{E}\left(\int_{0}^{t} \sigma^{2}(s) v_{s} d s\right)<\infty$, so the stochastic integral $\int_{0}^{t} \sigma(s) \sqrt{v_{s}} d B_{s}$ has zero Expectation. Using Fubini's theorem and the fundamental theorem of calculus, we obtain the forward equation

$$
\frac{\partial}{\partial t} \mathbb{E}\left(v_{t}\right)=\kappa\left(\theta(t)-\mathbb{E}\left(v_{t}\right)\right) .
$$

We can then re-arrange this equation to recover Eq 31 (note that $\sigma(t)$ does not affect $\mathbb{E}\left(v_{t}\right)$ ).

We now proceed along similar lines to Dufresne[Duf01]. Recall that $I_{t}=\int_{0}^{t} v_{s} d s$. By Itô's lemma, we have

$$
d\left(I_{t}^{2}\right)=2 I_{t} v_{t} d t
$$

Integrating and taking expectations, we obtain

$$
\begin{aligned}
\mathbb{E}\left(I_{t}^{2}\right)=2 \mathbb{E}\left(\int_{0}^{t} I_{s} v_{s} d s\right) & =2 \int_{0}^{t} \mathbb{E}\left(I_{s} v_{s}\right) d s \\
& =2 \int_{0}^{t} \int_{0}^{s} \mathbb{E}\left(v_{s} v_{u}\right) d u d s \\
& \leq 2 \int_{0}^{t} \int_{0}^{s} \mathbb{E}\left(v_{s}^{2}\right)^{\frac{1}{2}} \mathbb{E}\left(v_{u}^{2}\right)^{\frac{1}{2}} d u d s \\
& <\infty
\end{aligned}
$$

using Fubini's theorem and the Schwarz inequality. By the fundamental theorem of calculus, we see that

$$
\frac{\partial}{\partial t} \mathbb{E}\left(I_{t}^{2}\right)=2 \mathbb{E}\left(I_{t} v_{t}\right)
$$

We may repeat this procedure to compute $\mathbb{E}\left(I_{t} v_{t}\right)$ :

$$
I_{t} v_{t}=\int_{0}^{t}\left(I_{s} d v_{s}+v_{s} d I_{s}\right)=\int_{0}^{t} I_{s}\left(\kappa\left(\theta(s)-v_{s}\right) d s+\sigma(s) \sqrt{v_{s}} d B_{s}\right)+v_{s}^{2} d s .
$$

By the Schwarz inequality, we have that

$$
\mathbb{E}\left(I_{t}^{2} v_{t}\right) \leq \mathbb{E}\left(I_{t}^{4}\right)^{\frac{1}{2}} \mathbb{E}\left(v_{t}^{2}\right)^{\frac{1}{2}},
$$

and (by Jensen's inequality), we know that

$$
\frac{1}{t^{4}} \mathbb{E}\left(I_{t}^{4}\right)=\mathbb{E}\left(\left(\frac{1}{t} \int_{0}^{t} v_{s} d s\right)^{4}\right) \leq \mathbb{E}\left(\frac{1}{t} \int_{0}^{t} v_{s}^{4} d s\right)=\frac{1}{t} \int_{0}^{t} \mathbb{E}\left(v_{s}^{4}\right) d s
$$

and $\mathbb{E}\left(v_{s}^{4}\right)<\infty$ for $s \in[0, t]$ (see the Appendix). Thus $\mathbb{E}\left(I_{t}^{4}\right)<\infty, \mathbb{E}\left(v_{t}^{2}\right)<\infty, \mathbb{E}\left(I_{t}^{2} v_{t}\right)<\infty$ and

$$
\mathbb{E}\left(\int_{0}^{t} \sigma^{2}(s) I_{s}^{2} v_{s} d s\right)<\infty
$$

so we have sufficient integrability for the stochastic integral $\int_{0}^{t} \sigma(s) I_{s} \sqrt{v}_{s} d B_{s}$ in Eq 39 to have zero expectation. Thus we have the following forward equation for $\mathbb{E}\left(I_{t} v_{t}\right)$

$$
\frac{\partial}{\partial t} \mathbb{E}\left(I_{t} v_{t}\right)=\mathbb{E}\left(I_{t}\left(\kappa\left(\theta(t)-v_{t}\right)\right)+\mathbb{E}\left(v_{t}^{2}\right) .\right.
$$


Repeating the procedure again for $v_{t}^{2}$, we find that

$$
v_{t}^{2}=v_{0}^{2}+\int_{0}^{t} 2 v_{s}\left(\kappa\left(\theta(s)-v_{s}\right) d s+\sigma(s) \sqrt{v_{s}} d B_{s}\right)+\int_{0}^{t} \sigma^{2}(s) v_{s} d s .
$$

From the Appendix we have that $\mathbb{E}\left(v_{t}^{3}\right)<\infty$, so the Expectation of the stochastic integral in Eq 44 is also zero, and

$$
\frac{\partial}{\partial t} \mathbb{E}\left(v_{t}^{2}\right)=2 \mathbb{E}\left(v_{t} \kappa\left(\theta(t)-v_{t}\right)\right)+\sigma^{2}(t) \mathbb{E}\left(v_{t}\right) .
$$

Re-arranging this equation, we arrive at Eq (32).

\subsection{Numerical implementation}

Much like Dupire's[Dup94] forward equation for call options under a local volatility model, the equations in Theorem 2.1 look nice on paper, but are difficult to implement in practice because we have to compute higher order derivatives using finite derivatives with noisy/incomplete data. The following subsections address this problem by deriving Taylor series expansions for the expectation and the variance of the integrated variance.

\subsubsection{Small-time asymptotic behaviour}

Proposition 2.2 We have the following Taylor series expansions for the expectation and variance of $\frac{1}{t} \int_{0}^{t} v_{s} d s$ around $t=0$

$$
\begin{aligned}
\mathbb{E}\left(\frac{1}{t} \int_{0}^{t} v_{s} d s\right) & =v_{0}-\frac{1}{2} \kappa\left(v_{0}-\theta(0)\right) t+\frac{\kappa}{6}\left(\theta^{\prime}(0)-\kappa\left(\theta(0)-v_{0}\right)\right) t^{2}+\mathcal{O}\left(t^{3}\right) \\
\operatorname{Var}\left(\frac{1}{t} \int_{0}^{t} v_{s} d s\right) & =\frac{1}{3} v_{0} \sigma(0)^{2} t+\left(-\frac{1}{3} \kappa v_{0} \sigma(0)^{2}+\frac{1}{12} \kappa \theta(0) \sigma(0)^{2}+\frac{1}{6} v_{0} \sigma(0) \sigma^{\prime}(0)\right) t^{2}+\mathcal{O}\left(t^{3}\right) .
\end{aligned}
$$

Remark 2.1 For small-time asymptotics for call options and implied volatility under the Heston model, see Forde\&Jacquier[FJ09].

\subsubsection{A Bootstrapping procedure for piecewise linear $\theta(t)$ and $\sigma(t)$}

Proposition 2.3 Combining Eqs (27), (28) and (29), we have the following Taylor series expansions for the expectation and variance of $I_{t}$ around $s>0$ for $t>s$

$\mathbb{E}\left(I_{t}\right)=\mathbb{E}\left(I_{s}\right)+\mathbb{E}\left(v_{s}\right)(t-s)+\frac{\kappa}{2}\left[\theta(s)-\mathbb{E}\left(v_{s}\right)\right](t-s)^{2}+\frac{\kappa}{6}\left[\kappa \mathbb{E}\left(v_{s}\right)+\theta^{\prime}(s)-\kappa \theta(s)\right](t-s)^{3}+\mathcal{O}\left((t-s)^{4}\right)$, 


$$
\begin{aligned}
& \text { and } \\
& \qquad \begin{aligned}
& \operatorname{Var}\left(I_{t}\right) \\
= & \operatorname{Var}\left(I_{s}\right)+2\left[\mathbb{E}\left(I_{s} v_{s}\right)-\mathbb{E}\left(v_{s}\right) \mathbb{E}\left(I_{s}\right)\right](t-s) \\
+ & \left\{\mathbb{E}\left(v_{s}^{2}\right)-\mathbb{E}^{2}\left(v_{s}\right)-\kappa\left[\mathbb{E}\left(I_{s} v_{s}\right)-\mathbb{E}\left(I_{s}\right) \mathbb{E}\left(v_{s}\right)\right]\right\}(t-s)^{2} \\
+ & \left\{\frac{1}{3} \kappa^{2}\left[\mathbb{E}\left(I_{s} v_{s}\right)-\mathbb{E}\left(I_{s}\right) \mathbb{E}\left(v_{s}\right)\right]+\kappa\left[\mathbb{E}\left(v_{s}\right)^{2}-\mathbb{E}\left(v_{s}^{2}\right)\right]+\frac{\sigma^{2}(s)}{3} \mathbb{E}\left(v_{s}\right)\right\}(t-s)^{3} \\
+ & \frac{1}{12}\left\{\kappa^{3}\left[\mathbb{E}\left(I_{s}\right) \mathbb{E}\left(v_{s}\right)-\mathbb{E}\left(I_{s} v_{s}\right)\right]+2 \sigma(s) \sigma^{\prime}(s) \mathbb{E}\left(v_{s}\right)+\kappa \sigma^{2}(s)\left[\theta(s)-4 \mathbb{E}\left(v_{s}\right)\right]+7 \kappa^{2}\left(\mathbb{E}\left(v_{s}^{2}\right)-\mathbb{E}^{2}\left(v_{s}\right)\right)\right\}(t-s)^{4} \\
+ & \mathcal{O}\left((t-s)^{5}\right) .
\end{aligned}
\end{aligned}
$$

Proof. See Appendix B.

Corollary 2.4 We have the following Taylor series expansions for the expectation and variance of $\frac{1}{t} \int_{0}^{t} v_{s} d s$ around $t=0$

$$
\begin{aligned}
\mathbb{E}\left(\frac{1}{t} \int_{0}^{t} v_{s} d s\right) & =v_{0}-\frac{1}{2} \kappa\left(v_{0}-\theta(0)\right) t+\frac{\kappa}{6}\left(\theta^{\prime}(0)-\kappa\left(\theta(0)-v_{0}\right)\right) t^{2}+\mathcal{O}\left(t^{3}\right) \\
\operatorname{Var}\left(\frac{1}{t} \int_{0}^{t} v_{s} d s\right) & =\frac{1}{3} v_{0} \sigma(0)^{2} t+\left(-\frac{1}{3} \kappa v_{0} \sigma(0)^{2}+\frac{1}{12} \kappa \theta(0) \sigma(0)^{2}+\frac{1}{6} v_{0} \sigma(0) \sigma^{\prime}(0)\right) t^{2}+\mathcal{O}\left(t^{3}\right) .
\end{aligned}
$$

Proof. Follows from Proposition 2.3 for $t=0$.

In practice, we can extract the variance swap curve from log contract prices, and we could back out the term structure of the variance of the integrated variance using the Carr-Lee approximation in Eq (22), or we can choose to supply this exogenously. The following procedure outlines how one can use Propositions 2.2 and 2.3 to calibrate a time-dependent Heston model with piecewise linear $\theta(t)$ and $\sigma(t)$ :

- Choose a plausible value for $\kappa$.

- Fit the variance swap rate curve $\frac{1}{t} \mathbb{E}\left(\int_{0}^{t} v_{s} d s\right)$ from $t_{0}=0$ to $t_{1}>0$ with a quadratic, and the $\operatorname{Var}\left(\frac{1}{t} \int_{0}^{t} v_{s} d s\right)$ curve with a quadratic, whose leading order term is linear. Using Proposition 2.2 , we can then back out $v_{0}, \theta(0)$ and $\theta^{\prime}(0)$ from the coefficients of the first quadratic, and $\sigma(0)$ and $\sigma^{\prime}(0)$ from the coefficients of the second quadratic.

- Using the values for $\theta(0), \theta^{\prime}(0), \sigma(0)$ and $\sigma^{\prime}(0)$, construct affine functions for $\theta(t)$ and $\sigma(t)$ over $\left[0, t_{1}\right]$.

- We then solve Eqs $(27),(28)$ and $(29)$ analytically over $\left[0, t_{1}\right]$, so as to compute $\mathbb{E}\left(v_{t_{1}}^{2}\right), \mathbb{E}\left(I_{t_{1}} v_{t_{1}}\right)$, $\mathbb{E}\left(I_{t_{1}}^{2}\right)$, and hence $\operatorname{Var}\left(I_{t_{1}}^{2}\right)$.

- Ignore the $\mathcal{O}\left((t-s)^{4}\right)$ term in Eq (48) and the $\left.\mathcal{O}(t-s)^{5}\right)$ term in Eq (49). Then, from pre-specified values for $\mathbb{E}\left(I_{t_{2}}\right)$ and $\operatorname{Var}\left(I_{t_{2}}\right)$ with $t_{2}>t_{1}$, back out $\theta^{\prime}\left(t_{1}\right)$ and $\sigma^{\prime}\left(t_{1}\right)$ using Eqs (48) and (49) respectively with $s=t$ and $t=t_{2}$. We then use these derivatives to construct affine functions for $\theta(t)$ and $\sigma(t)$ over $\left[t_{1}, t_{2}\right]$, so that $\theta(t)$ and $\sigma(t)$ are piecewise linear (and continuous) over $\left[0, t_{2}\right]$.

- Repeat this procedure. 


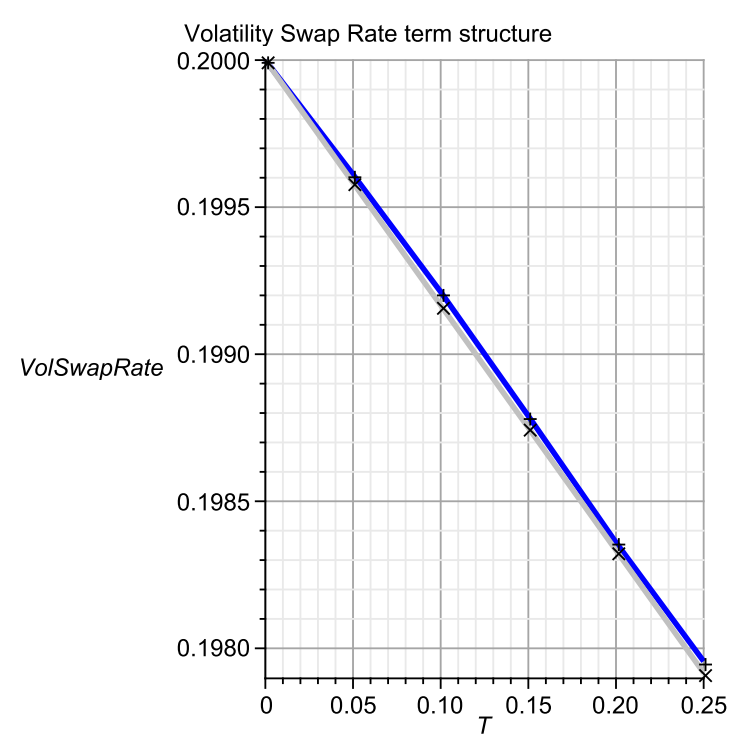

Figure 2: Here we have plotted the volatility swap term structure for a time-dependent Heston model with parameters $\kappa=1.15, \theta(t)=.04-.005 t, \sigma(t)=.2-.05 t, y_{0}=.04$, using an Euler Monte Carlo scheme with 500,000 simulations and 5000 time steps (blue) versus the series approximation in Eq 52 (grey).

\subsubsection{Small-time approximation for the volatility swap rate}

Combining Proposition 2.2 with the Brockhaus-Long approximation in Eq 20, we obtain the following approximation for the volatility swap rate

$$
\begin{aligned}
\mathbb{E}\left(\left(\frac{1}{t} \int_{0}^{t} v_{s} d s\right)^{\frac{1}{2}}\right) \approx & \sqrt{v_{0}}+\frac{1}{\sqrt{v_{0}}}\left[\frac{1}{4} \kappa\left(\theta(0)-v_{0}\right)-\frac{1}{24} \sigma(0)^{2}\right] t \\
& +\left[\frac{\sigma(0)^{2}}{32 v_{0}^{\frac{3}{2}}} \kappa\left(\theta(0)-v_{0}\right)-\frac{1}{8 v_{0}^{\frac{3}{2}}}\left(-\frac{1}{3} v_{0} \kappa \sigma(0)^{2}+\frac{1}{12} \kappa \theta(0) \sigma(0)^{2}+\frac{1}{6} v_{0} \sigma(0) \sigma^{\prime}(0)\right)\right. \\
& \left.+v_{0}^{\frac{1}{2}}\left(\frac{1}{12 v_{0}}\left(\kappa \theta^{\prime}(0)-\kappa^{2} \theta(0)+\kappa^{2} v_{0}\right)-\frac{1}{576 v_{0}^{2}} \kappa\left(\theta(0)-v_{0}\right)^{2}\right)\right] t^{2}
\end{aligned}
$$

From Eq (51), we see that this truncated Taylor series approximation will work better for $\sigma^{2}(0) t \ll$ 1 (see also Figure 2 above).

\section{References}

[AP07] Andersen, L.B.G., and V.V.Piterbarg, "Moment Explosions in Stochastic Volatility Models", Finance and Stochastics, 2007.

[BL00] Brockhaus, O., and D.Long, "Volatility Swaps made simple", Risk, January 2000. 
[BL78] Breeden, D. and R. Litzenberger, "Prices of state contingent claims implicit in options prices", Journal of Business, 51:621-651, 1978.

[Buehler06] Buehler, H., "Volatility markets: consistent modelling, hedging and practical implementation", PhD dissertation, 2006.

[CGMY03] Carr, P., H.Geman, D.Madan and M.Yor, "Stochastic volatility for Lévy processes", Mathematical Finance, Vol. 13 No. 3, pp. 345-382, July 2003.

[CL08] Carr, P. and R.W.Lee, "Robust Replication of Volatility Derivatives", working paper, 2008.

[CS00] P.Carr and M. Schroder, "On the Valuation of Arithmetic-Average Asian Options: The GemanYor Laplace Transform Revisited", Preprint, Mannheim and New York, 2000.

[Duf01] Dufresne, D. "The integrated square-root process". Research Paper no. 90, Centre for Actuarial Studies, University of Melbourne, 2001.

[Dup94] Dupire, B., "Pricing with a smile", Risk, 7:1820, January 1994.

[Gath07] Gatheral, J., "Developments in Volatility Derivatives pricing", ICBI Global Derivatives and Risk Management conference, Paris, May 2007.

[GY93] Geman, H. and M.Yor, "Bessel processes, Asian options and perpetuities", Mathematical Finance, vol. 4, no. 3, pp. 349-375, 1993.

[DGY01] Donati-Martin, C., R.Ghomasni and M.Yor., "On certain Markov processes attached to exponential functionals of Brownian motion", Applications to Asian options, Revista Matematica Iberoamericana Madrid,(1) 179-193, 2001.

[FJ09] Forde, M., A.Jacquier, "Small-time asymptotics for Implied volatility under the Heston model", forthcoming in the International Journal of Theoretical and Applied Finance, 2009.

[Hest93] Heston, S., "A closed-form solution for options with stochastic volatility with applications to bond and currency options", Review of Financial Studies, 1993.

[HW87] Hull, J. and A.White, "The pricing of options on assets with stochastic volatilities", J.Finance, 42, 281-299, 1987, 17.

[HK08] Hurd, T.R. and A.Kuznetsov, "Explicit formulas for Laplace transforms of stochastic integrals", Markov Processes and Related Fields, 14, 277-290, 2008.

[KS91] Karatzas, I. and S.Shreve, "Brownian motion and Stochastic Calculus", Springer-Verlag, 1991.

[Levy92] Levy, E.,"Pricing European average rate currency options", Journal of International Money and Finance, vol. 11, pp. 474-491, 1992.

[MP98] Milevsky, M.A. and S.E.Posner, "Asian options, the sum of lognormals, and the reciprocal gamma distribution", Journal of Financial and Quantitative Analysis, vol. 33, no. 3, pp. 409-422, 1998.

[Neu92] Neuberger, A. "Volatility Trading", London Business School WP, 1992. 
[Pit06] Piterbarg, V. "Time to Smile", Risk, 2006.

[PM98] Posner.,S.E. and M.A.Milevsky, "Valuing exotic options by approximating the spd with higher moments", Journal of Financial Engineering, vol. 7, no. 2, pp. 109-125, 1998.

[RS95] Rogers, L.C.G. and Z.Shi, The value of an Asian option, Journal of Applied Probability, no. 32, pp. 1077-1088, 1995.

[RY91] Revuz, D. and M.Yor, "Continuous Martingales and Brownian motion", Springer, 1991.

[T99] Thompson, G.W.P., "Topics in Mathematical Finance", PhD thesis, University of Cambridge, 1999.

\section{A The time-dependent Heston process as a time-changed Bessel process}

Consider the following time-dependent extension of a standard Bessel squared process, which solves the following SDE

$$
d\left(R_{t}^{2}\right)=2 R_{t} d B_{t}+\delta(t) d t
$$

with $\delta(t)$ time-dependent. Now consider

$$
v_{t}=e^{-\kappa t} R_{\int_{0}^{t} \frac{1}{4} \sigma^{2}(s) e^{\kappa s} d s} .
$$

Then

$$
d v_{t}=-\kappa v_{t}+\frac{1}{4} \delta(t) \sigma^{2}(t) d t+\sigma(t) \sqrt{v_{t}} d B_{t}
$$

If we set

$$
\delta(t)=\frac{4 \kappa \theta(t)}{\sigma^{2}(t)},
$$

then $v_{t}$ satisfies the same SDE as the time-dependent Heston model

$$
d v_{t}=\kappa\left(\theta(t)-v_{t}\right) d t+\sigma(t) \sqrt{v_{t}} d B_{t} .
$$

for which we have strong uniqueness. Using the comparison thereom (Proposition 2.18 on page 293 in Karatzas\&Shreve[KS91]), we have that

$$
\mathbb{P}\left(R_{t} \leq R_{t}^{(1)}, 0 \leq t \leq \infty\right)=1,
$$

where $R^{(1)}$ is a standard Bessel squared process $B E S Q^{\delta_{\max }}$, with dimension $\delta_{\max }=\max _{0 \leq t \leq T} \delta(u)$. The transition density for the $B E S Q^{\delta_{\max }}$ semigroup is well known (see e.g. Revuz\&Yor[RY91]), and $R_{t}^{1}$ has finite moments of all order $0 \leq t<\infty$. Thus we conclude that

$$
\mathbb{E}\left(R_{t}^{8}\right)<\infty,
$$

and

$$
\mathbb{E}\left(v_{t}^{4}\right)<\infty .
$$




\section{B Proof of Proposition 2.3}

Let $t \geq 0$. Solving the ODE in Eq (35), and expanding as a Taylor series around $t=s$ and integrating gives

$$
\begin{aligned}
\mathbb{E}\left(I_{t}\right) & =\mathbb{E}\left(I_{s}\right)+\mathbb{E}\left(v_{s}\right)(t-s)+\frac{\kappa}{2}\left[\theta(s)-\mathbb{E}\left(v_{s}\right)\right](t-s)^{2} \\
& +\frac{\kappa}{6}\left[\kappa \mathbb{E}\left(v_{s}\right)+\theta^{\prime}(s)-\kappa \theta(s)\right](t-s)^{3}+\frac{\kappa}{24}\left[\kappa^{2}\left(\theta-\mathbb{E}\left(v_{s}\right)\right)+\theta^{\prime \prime}(s)-\kappa \theta^{\prime}(s)\right](t-s)^{4}+\mathcal{O}\left((t-s)^{5}\right),
\end{aligned}
$$

and

$$
\begin{aligned}
\mathbb{E}\left(I_{t}\right)^{2} & =\mathbb{E}\left(I_{s}\right)^{2}+2 \mathbb{E}\left(I_{s}\right) \mathbb{E}\left(v_{s}\right)(t-s)+\left[\kappa \theta(s) \mathbb{E}\left(I_{s}\right)+\mathbb{E}^{2}\left(v_{s}\right)-\kappa \mathbb{E}\left(v_{s}\right) \mathbb{E}\left(I_{s}\right)\right](t-s)^{2} \\
& +\kappa\left[\left(\frac{\kappa \mathbb{E}\left(I_{s}\right)}{3}-\mathbb{E}\left(v_{s}\right)\right)\left(\mathbb{E}\left(v_{s}\right)-\theta(s)\right)+\frac{1}{3} \mathbb{E}\left(I_{s}\right) \theta^{\prime}(s)\right](t-s)^{3}+\Psi(s, t)(t-s)^{4}+\mathcal{O}\left((t-s)^{5}\right),
\end{aligned}
$$

where

$$
\begin{aligned}
\Psi(s, t) & =\frac{\kappa}{12}\left\{\kappa^{2} \mathbb{E}\left(I_{s}\right)\left[\theta(s)-\mathbb{E}\left(v_{s}\right)\right]+\mathbb{E}\left(I_{s}\right)\left[\theta^{\prime \prime}(s)-\kappa \theta^{\prime}(s)\right]+7 \kappa \mathbb{E}^{2}\left(v_{s}\right)\right\} \\
& -\frac{5}{6} \mathbb{E}\left(v_{s}\right) \kappa^{2} \theta(s)+\frac{1}{3} \mathbb{E}\left(v_{s}\right) \kappa \theta^{\prime}(s)+\frac{1}{4} \kappa^{2} \theta(s)^{2} .
\end{aligned}
$$

We also have

$$
\begin{aligned}
\mathbb{E}\left(v_{t}^{2}\right) & =\mathbb{E}\left(v_{s}^{2}\right)+\left[-2 \kappa \mathbb{E}\left(v_{s}^{2}\right)+\mathbb{E}\left(v_{s}\right) \sigma^{2}(s)+2 \kappa \theta(s) \mathbb{E}\left(v_{s}\right)\right](t-s) \\
& +\left\{\kappa \theta(s)\left[\kappa \theta(s)+\frac{\sigma^{2}(s)}{2}\right]+\mathbb{E}\left(v_{s}\right)\left[\sigma(s) \sigma^{\prime}(s)-\frac{3 \kappa}{2} \sigma^{2}(s)+\kappa \theta^{\prime}(s)-3 \kappa^{2} \theta(s)\right]+2 \kappa^{2} \mathbb{E}\left(v_{s}^{2}\right)\right\}(t-s)^{2} \\
& +\Upsilon(s, t)(t-s)^{3}+\mathcal{O}\left((t-s)^{4}\right)
\end{aligned}
$$

where

$$
\begin{gathered}
\Upsilon(s, t)=-\frac{4 \kappa^{3}}{3} \mathbb{E}\left(v_{s}^{2}\right)+\frac{7}{6} \sigma^{2}(s) \mathbb{E}\left(v_{s}\right) \kappa^{2}-\kappa^{3} \theta^{2}(s)+\frac{1}{3} \mathbb{E}\left(v_{s}\right) \sigma^{\prime 2}(s)-\frac{4}{3} \kappa \sigma(s) \sigma^{\prime}(s) \mathbb{E}\left(v_{s}\right) \\
+\frac{7}{3} \kappa^{3} \theta(s) \mathbb{E}\left(v_{s}\right)-\frac{1}{2} \sigma^{2}(s) \kappa^{2} \theta(s)+\frac{1}{6} \sigma^{2}(s) \kappa \theta^{\prime}(s)+\kappa^{2} \theta(s) \theta^{\prime}(s)+\frac{1}{3} \mathbb{E}\left(v_{s}\right) \kappa \theta^{\prime \prime}(s) \\
+\frac{1}{3} \mathbb{E}\left(v_{s}\right) \sigma(s) \sigma^{\prime \prime}(s)+\frac{2}{3} \sigma(s) \sigma^{\prime}(s) \kappa \theta(s)-\frac{4}{3} \kappa^{2} \theta^{\prime}(s) \mathbb{E}\left(v_{s}\right),
\end{gathered}
$$

and

$$
\begin{aligned}
\mathbb{E}\left(I_{t} v_{t}\right) & =\mathbb{E}\left(I_{s} v_{s}\right)+\left[\kappa \theta(s) \mathbb{E}\left(I_{s}\right)-\kappa \mathbb{E}\left(I_{s} v_{s}\right)+\mathbb{E}\left(v_{s}^{2}\right)\right](t-s) \\
& +\frac{1}{2}\left[\kappa^{2}\left(\mathbb{E}\left(I_{s} v_{s}\right)-\theta(s) \mathbb{E}\left(I_{s}\right)\right)+3 \kappa\left(\mathbb{E}\left(v_{s}\right) \theta(s)-\mathbb{E}\left(v_{s}^{2}\right)\right)+\sigma(s)^{2} \mathbb{E}\left(v_{s}\right)+\kappa \theta^{\prime}(s) \mathbb{E}\left(I_{s}\right)\right](t-s)^{2} \\
& +\Xi(s, t)(t-s)^{3}+\mathcal{O}\left((t-s)^{4}\right),
\end{aligned}
$$

where

$$
\begin{aligned}
\Xi(s, t) & =\frac{1}{6}\left\{\kappa^{3}\left[\theta(s) \mathbb{E}\left(I_{s}\right)-\mathbb{E}\left(I_{s} v_{s}\right)\right]+4 \kappa\left(\theta^{\prime}(s)-\sigma^{2}(s)\right) \mathbb{E}\left(V_{s}\right)+\kappa\left[\theta(s) \sigma^{2}(s)-\kappa \theta^{\prime}(s) \mathbb{E}\left(I_{s}\right)\right]\right\} \\
& +\frac{1}{6}\left\{2 \mathbb{E}\left(v_{s}\right) \sigma(s) \sigma^{\prime}(s)-\kappa^{2} \theta(s)\left[10 \mathbb{E}\left(v_{s}\right)-3 \theta(s)\right]+\kappa \theta^{\prime \prime}(s) \mathbb{E}\left(I_{s}\right)+7 \kappa^{2} \mathbb{E}\left(v_{s}^{2}\right)\right\}
\end{aligned}
$$


Thus we obtain

$$
\begin{aligned}
\mathbb{E}\left(I_{t}^{2}\right) & =2 \int_{0}^{t} \mathbb{E}\left(I_{u} v_{u}\right) d u \\
& =2 \int_{0}^{s} \mathbb{E}\left(I_{u} v_{u}\right) d u+2 \int_{s}^{t} \mathbb{E}\left(I_{u} v_{u}\right) d u \\
& =\mathbb{E}\left(I_{s}^{2}\right)+2 \mathbb{E}\left(I_{s} v_{s}\right)(t-s)+\left[\kappa \theta(s) \mathbb{E}\left(I_{s}\right)-\kappa \mathbb{E}\left(I_{s} v_{s}\right)+\mathbb{E}\left(v_{s}^{2}\right)\right](t-s)^{2} \\
& +\frac{1}{3}\left[\kappa^{2}\left(\mathbb{E}\left(I_{s} v_{s}\right)-\theta(s) \mathbb{E}\left(I_{s}\right)\right)+3 \kappa\left(\mathbb{E}\left(v_{s}\right) \theta(s)-\mathbb{E}\left(v_{s}^{2}\right)\right)+\sigma^{2}(s) \mathbb{E}\left(v_{s}\right)+\kappa \theta^{\prime}(s) \mathbb{E}\left(I_{s}\right)\right](t-s)^{3} \\
& +\frac{1}{2} \Xi(s, t)(t-s)^{4} \\
& +\mathcal{O}\left((t-s)^{5}\right) .
\end{aligned}
$$

Therefore

$$
\begin{aligned}
\operatorname{Var}\left(\int_{0}^{t} v_{u} d u\right) & =\left[\mathbb{E}\left(I_{s}^{2}\right)-\mathbb{E}^{2}\left(I_{s}\right)\right]+2\left[\mathbb{E}\left(I_{s} v_{s}\right)-\mathbb{E}\left(v_{s}\right) \mathbb{E}\left(I_{s}\right)\right](t-s) \\
& +\left\{\mathbb{E}\left(v_{s}^{2}\right)-\mathbb{E}^{2}\left(v_{s}\right)-\kappa\left[\mathbb{E}\left(I_{s} v_{s}\right)-\mathbb{E}\left(I_{s}\right) \mathbb{E}\left(v_{s}\right)\right]\right\}(t-s)^{2} \\
& +\left\{\frac{1}{3} \kappa^{2}\left[\mathbb{E}\left(I_{s} v_{s}\right)-\mathbb{E}\left(I_{s}\right) \mathbb{E}\left(v_{s}\right)\right]+\kappa\left[\mathbb{E}^{2}\left(v_{s}\right)-\mathbb{E}\left(v_{s}^{2}\right)\right]+\frac{\sigma^{2}(s)}{3} \mathbb{E}\left(v_{s}\right)\right\}(t-s)^{3} \\
& +\left\{\kappa^{3}\left[\mathbb{E}\left(I_{s}\right) \mathbb{E}\left(v_{s}\right)-\mathbb{E}\left(I_{s} v_{s}\right)\right]+2 \sigma(s) \sigma^{\prime}(s) \mathbb{E}\left(v_{s}\right)+\kappa \sigma^{2}(s)\left[\theta(s)-4 \mathbb{E}\left(v_{s}\right)\right]+7 \kappa^{2}\left(\mathbb{E}\left(v_{s}^{2}\right)-\mathbb{E}^{2}\left(v_{s}\right)\right)\right\} \frac{(t-s)^{4}}{12} \\
& +\mathcal{O}\left((t-s)^{5}\right) .
\end{aligned}
$$

Now, take $s=0$, then $\mathbb{E}\left(I_{s}\right)=0, \mathbb{E}\left(v_{s}\right)=v_{0}$ and $\mathbb{E}\left(I_{s} v_{s}\right)=\mathbb{E}\left(I_{s}\right) \mathbb{E}\left(v_{s}\right)=0$, so we have

$$
\operatorname{Var}\left(\int_{0}^{t} v_{u} d u\right)=\frac{1}{3} \sigma^{2}(0) v_{0} t^{3}+\frac{1}{6} \sigma(0) \sigma^{\prime}(0) v_{0}+\frac{\kappa}{12} \sigma^{2}(0)\left[\theta(0)-4 v_{0}\right] t^{4}+\mathcal{O}\left(t^{5}\right) .
$$

